\title{
Enjeux théoriques et opérationnels de l'analyse économique du système judiciaire
}

\author{
Ludivine Roussey
}

\section{(2) OpenEdition}

Édition électronique

URL : http://journals.openedition.org/ei/5692

DOI : 10.4000/ei.5692

ISSN : 2553-1891

\section{Éditeur}

Association Économie et Institutions

Référence électronique

Ludivine Roussey, «Enjeux théoriques et opérationnels de l'analyse économique du système judiciaire », Économie et institutions [En ligne], 24 | 2016, mis en ligne le 30 juin 2016, consulté le 01 mai 2019 URL : http://journals.openedition.org/ei/5692 ; DOI : 10.4000/ei.5692

Ce document a été généré automatiquement le 1 mai 2019.

Revue Économie et institutions 


\section{Enjeux théoriques et opérationnels de l'analyse économique du système judiciaire}

Ludivine Roussey

\section{Introduction}

1 La judiciarisation croissante, l'engorgement des juridictions et les délais de traitement des affaires perçus comme trop longs sont autant de manifestations de la crise que subissent les institutions judiciaires à travers le monde. Dans un tel contexte, et en période de forte contrainte budgétaire, savoir comment le fonctionnement du système judiciaire peut répondre au mieux aux besoins des justiciables et, dans une perspective plus large, aux citoyens, relève d'enjeux cruciaux.

2 En pratique, l'urgence des situations de nombreux systèmes judiciaires encourage les discussions de la part des instances internationales, des responsables politiques et des professionnels du droit sur les dysfonctionnements de la justice et la mesure de la performance judiciaire. En France, des changements organisationnels ont été impulsés par la mise en application de la Loi organique sur les lois de finance (LOLF), votée en 2001, qui modifie les règles budgétaires et comptables dans le but affiché de permettre une meilleure efficacité de la justice. En Europe, la Commission Européenne Pour l'Efficacité de la Justice (CEPEJ) est devenue, depuis sa création en 2002, un observatoire des systèmes judiciaires européens et fournit, avec la publication d'un rapport d'évaluation bisannuel, un outil d'information et de comparaison de l'efficacité des systèmes. Au niveau mondial, le programme Doing Business, initié en 2002, évalue, entre autres, les normes et les procédures relatives à la règlementation des affaires et encourage ainsi des réformes permettant aux entrepreneurs de « faire des affaires » plus facilement dans certains pays.

3 Si ces expériences et leurs implications en termes de réformes mettent en lumière des aspects certes importants de l'organisation de la justice, force est de constater qu'elles n'abordent pas la question du fonctionnement des institutions judiciaires de manière 
pleinement satisfaisante et ce pour trois raisons. Elles échouent tout d'abord à prendre en compte les deux principaux objectifs du système judiciaire, à savoir : la résolution des litiges de manière rapide et équitable et l'incitation des individus à l'adoption de comportements socialement désirables. Ensuite, elles ne considèrent pas de manière systématique les contraintes que représentent la demande de justice et les ressources pouvant être consacrées au système judiciaire. Enfin, elles n'envisagent que marginalement les interactions entre le fonctionnement de la justice, les contraintes du système judiciaire et les résultats directs et indirects de son activité.

4 Pourtant, l'évaluation du système judiciaire et l'anticipation de ses effets sur les comportements individuels des justiciables et des praticiens du droit (avocats, magistrats, etc.) ne peut être cohérente sans la prise en compte de l'ensemble de ces facteurs. L'analyse économique, parce qu'elle offre des outils (théorie des jeux, théorie des incitations, économétrie etc.) et des méthodes adaptées (calcul d'optimisation sous contrainte par exemple), permet une compréhension fine du fonctionnement du système judiciaire, des contraintes auxquelles il est soumis, et de ses effets non seulement sur la qualité du service rendu aux justiciables mais aussi sur les comportements individuels.

5 L'analyse économique du droit débute en 1960 avec l'article de Coase. Dans The Social cost, Coase indique que les institutions, parmi lesquelles les institutions juridiques et judiciaires, ne sont pas neutres vis-à-vis des échanges économiques en présence de coûts de transaction. L'auteur met ainsi en évidence l'effet incitatif des règles de droit sur le comportement des agents économiques. Il révèle alors l'intérêt des dispositifs juridiques pour corriger les externalités et atteindre l'efficacité économique. Le théorème de Coase a inspiré de nombreux économistes et juristes qui ont cherché depuis à comprendre comment les prix implicites contenus dans les règles de droit pouvaient affecter les choix des individus et leur manière d'interagir les uns avec les autres. Un très grand nombre de domaines du droit ont été étudiés, en droit civil, privé ou pénal : le droit des contrats, le droit de la responsabilité, le droit de la famille, le droit de la concurrence... Alors que les effets des règles de droit sur les comportements économiques et sociaux sont extensivement étudiés en économie du droit, la littérature économique sur les modalités organisationnelles de la mise en œuvre du droit - c'est-à-dire sur le fonctionnement du système judiciaire - est plus embryonnaire.

6 Les développements récents portants sur les conséquences de certaines modalités du fonctionnement de la justice sur les décisions des individus ou sur le bien-être social offrent quelques illustrations de l'apport de l'analyse économique au débat sur la performance de la justice. Ils montrent aussi tout l'intérêt de l'analyse économique du droit pour pouvoir anticiper les répercussions de réformes organisationnelles ou budgétaires sur le système judiciaire lui-même et plus généralement sur l'économie.

7 Cet article est organisé de la manière suivante. Dans la deuxième section, nous dresserons un état des lieux de la crise du système judiciaire en nous basant sur le cas français. Dans la section suivante, nous proposerons une analyse critique des programmes d'évaluation des systèmes judiciaires conduits en France, en Europe et internationalement. La quatrième section proposera un nouveau cadre d'analyse du fonctionnement du système judiciaire. Enfin, la dernière section explorera ce cadre en montrant comment l'économie peut contribuer à l'analyse du fonctionnement du système judiciaire, au travers de contributions variées en analyse économique du droit. 


\section{Etat des lieux}

Dans une perspective économique, la justice peut être abordée comme un marché où l'offre correspond aux ressources fournies par l'Etat et la demande est une demande de décisions de justice (Deffains et Doriat-Duban, 2001, p. 952). Le déséquilibre du marché de la justice, qui s'observe dans beaucoup d'économies modernes, s'explique par l'évolution « en ciseaux » de l'offre et de la demande. L'excédent de demande de justice par rapport à l'offre a pour conséquence une détérioration de la qualité, au point que l'on parle aujourd'hui de « crise » de la justice

En l'espace d'une décennie, malgré l'accroissement des budgets en valeur absolue, les moyens se sont raréfiés au regard de l'accroissement des besoins à satisfaire en matière de justice. Alors même que les gouvernements successifs semblent vouloir faire de la justice une priorité budgétaire, les revendications des professionnels de justice et des citoyens sur le thème de l'insuffisance des moyens de la justice sont permanentes. Ces revendications semblent toucher de nombreux pays. Pour mieux comprendre cette situation, nous proposons d'étudier le cas de la France, comme un cas d'école.

$\mathrm{Au}$ cours des années $2000^{1}$, le budget français de la justice a connu une hausse progressive d'environ $4 \%$ par an. Mais cette croissance apparente des moyens alloués à la justice doit être relativisée de plusieurs manières.

11 Tout d'abord, l'augmentation du budget est absorbée par une part des crédits de plus en plus importante accordée à l'administration pénitentiaire, dont les besoins sont par ailleurs immenses. Les crédits de paiements consacrés au programme «administration pénitentiaire » représentaient $36 \%$ de la mission Justice en 2006. Ils s'élèvent à 50,4 \% en 2012. Cette tendance s'opère au détriment d'autres programmes de la mission Justice, comme l'accès au droit et à la justice.

De plus, historiquement, le retard accumulé dans les moyens consacrés à la justice est immense. Dans une analyse approfondie du budget de la justice en France de la Restauration au seuil du Xxi ${ }^{e}$ siècle, Asselain (2009) met en évidence l'appauvrissement brutal de la justice entre le début de la III ${ }^{e}$ République et la réforme de la magistrature de 1958. Par contraste avec cette période de déclin, le redressement qui s'est amorcé dans les années 70, parait tout à la fois de grande ampleur et prolongé, mais il est en fait tout juste suffisant pour retrouver les niveaux du milieu du xix ${ }^{e}$ siècle. Cette évolution en « V » du budget s'observe en analysant divers indicateurs, tels que la part des dépenses de justice dans le budget général de l'Etat (figure 1), dans le PIB, ou encore la part de la justice dans les effectifs de la fonction publique d'Etat. 
Figure 1. Part des dépenses de Justice dans le budget de l'Etat (en pourcentage) de 1822 à 2012

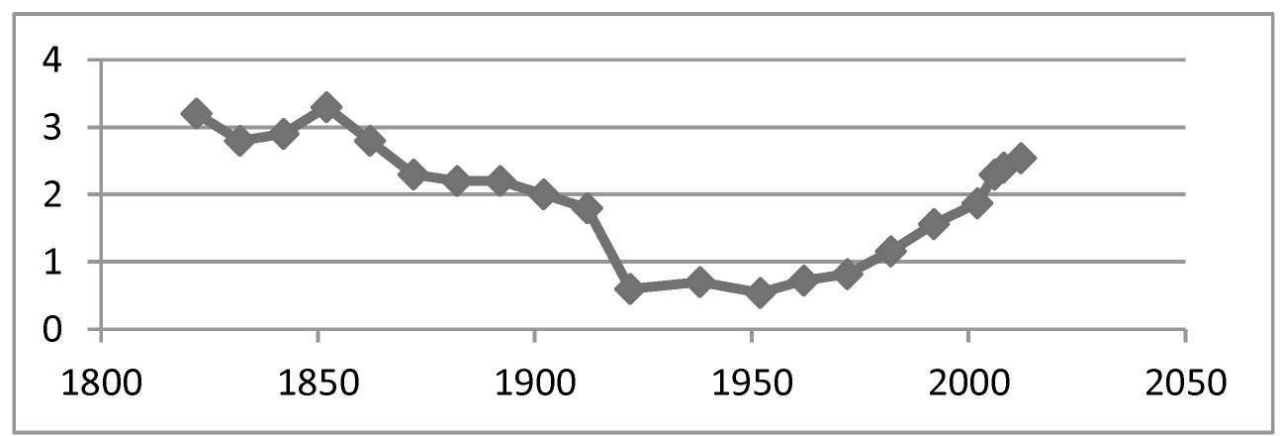

Source : Jean-Charles Asselain [2009] p. 158 et Ministère de l'Economie et des Finances (CEPEJ, 2014, p.60), le budget alloué aux tribunaux, au ministère public et à l'aide judiciaire, en pourcentage du PIB, place la France au $37^{\mathrm{e}}$ rang sur 45 pays membres du Conseil de l'Europe en 2008. Alors que la croissance du budget entre 2010 et 2012 s'élève à $5 \%$ en moyenne en Europe, en France, elle n'est que de $2 \%$. Comparée à 15 pays d'Europe occidentale de richesse similaire ${ }^{2}$, la France semble encore plus en retard du point de vue des moyens consacrés au système judiciaire. Elle occupe systématiquement le bas des classements, qu'il s'agisse du budget rapporté à la population ou à la richesse nationale du nombre de juges, du nombre de procureurs ou encore du nombre de fonctionnaires de justice (Jean, 2010).

Enfin, de 2002 à 2008, le nombre d'affaires civiles a augmenté de $58 \%$, les décisions en matière pénale de $10 \%$, et le taux de réponse pénale est passé de $68 \%$ à $85 \%$. Parallèlement, le budget n'a augmenté que de $5 \%^{3}$. En l'espace de quelques dizaines d'années seulement, les attentes vis-à-vis de la justice, et donc les missions de l'institution, se sont considérablement élargies. Les relations économiques et sociales devenant de plus en plus impersonnelles et complexes, les institutions informelles ne suffisent plus à encadrer les nouveaux modes d'échanges qui se diffusent. Du coté des nouvelles technologies, le rythme des innovations est tellement rapide qu'il laisse les individus démunis face aux risques que cela peut représenter. Le temps et le discernement faisant défaut pour pouvoir réglementer en amont, la priorité est souvent laissée aux libertés individuelles. La justice est sollicitée en dernier ressort lorsque des dysfonctionnements surviennent. Ce mode de régulation a posteriori des comportements sociaux donne lieu à ce que l'on nomme aujourd'hui l'« hyperjudiciarisation ». Dans son rapport de 2010, la CEPEJ offre un résumé simple et clair des conséquences de la double dynamique de baisse relative de l'offre et de demande croissante : « La France note [...] un effet de ciseaux sur le budget de la justice judiciaire, entre une évolution négative ou stagnante des crédits et une évolution croissante des besoins, qu'il s'agisse des personnels, des crédits de fonctionnement ou des frais de justice. » (CEPEJ, 2010, p. 51). Cette tendance, observée depuis plusieurs dizaines d'années, comme en témoignent de manière récurrente les rapports de la Commission des lois, de la Commission des finances ou encore de la Cour des comptes, s'est accélérée avec la crise économique et budgétaire récente. En effet, la crise de 2007-2008 a eu pour effet d'accroître les besoins du côté de la demande de justice et, du côté de l'offre, de renforcer la concurrence budgétaire entre les différentes missions de l'Etat. L'insuffisance des moyens, comparée aux besoins, tend non seulement à désorganiser les juridictions mais aussi à rendre difficile la mise en œuvre de 
réformes organisationnelles qui pourraient permettre, à terme, d'améliorer l'efficience de la justice. En effet, certaines réformes pourtant jugées nécessaires et acceptées unanimement par les différents acteurs, soit s'avèrent très complexes à conduire en raison des moyens matériels et humains importants qu'elles nécessitent (par exemple la carte judiciaire), soit sont mises en échec par le manque de ressources (par exemple la collégialité de l'instruction.

Le déséquilibre du marché de la justice a pour conséquence des dysfonctionnements qui conduisent petit à petit à une dégradation de nombreux aspects de la qualité de la justice. Nous en donnons ici deux exemples à travers les délais de traitement des affaires dus à l'engorgement des juridictions et la perte de confiance de l'opinion publique.

L'une des garanties d'un procès équitable selon l'Article 6 de la Convention Européenne des Droits de l'Homme est qu'une cause soit entendue dans un « délai raisonnable ». De 1959 à 2010 la France a ainsi été condamnée 279 fois pour violation de cette obligation. Dans les études internationales comme celles menées par la CEPEJ, les délais de jugement sont un indicateur de référence permettant d'évaluer la qualité de la justice. La CEPEJ calcule le taux de variation du stock d'affaires pendantes (clearance rate) en divisant le nombre d'affaires terminées par le nombre d'affaires nouvelles et en multipliant le résultat par 100. En France, en 2012, le taux de variation du stock d'affaires civiles et commerciales contentieuses en première instance était inférieur à 100 . Cela indique que la France ne parvient pas tout à fait à faire face aux flux d'affaires pendantes (malgré une légère amélioration depuis 2006 de 1,1\%), d'où une augmentation de l'arriéré judiciaire (CEPEJ, 2014, p. 212). Au contraire, des pays comme l'Autriche, l'Allemagne, la Finlande, le Danemark, ou l'Italie parviennent à réduire leur arriéré judiciaire grâce à des taux de variation de stock de plus de $100 \%$. Toujours selon la CEPEJ, le nombre estimé de jours nécessaires pour résoudre les affaires civiles contentieuses devant les tribunaux de première instance était de 311 jours en 2012, soit plus qu'en Suisse (127), en Autriche (135), en Norvège (160), au Danemark (165), en Allemagne (183), en Pologne (195) ou en Espagne (264). Si l'on se réfère aux annuaires statistiques de la Justice, le constat n'est pas moins frappant: la durée moyenne de traitement des affaires civiles, toutes nature de contentieux confondues, est passée de 8,7 mois en 2006 à 9,3 mois en 2010 devant les tribunaux de grande instance, de 5,1 à 5,6 mois devant les tribunaux d'instance et les tribunaux paritaires des baux ruraux, de 12,4 à 13,7 mois devant les conseils de prud'hommes et de 2,6 à 5,9 mois devant les tribunaux de commerce. Dans le domaine pénal, les difficultés se situent principalement au niveau du Ministère Public, puisque, comparativement aux autres pays européens, les procureurs français sont à la fois les moins nombreux (moins de 3 procureurs pour 100000 habitants en 2012) et les plus sollicités (2758 affaires reçues par procureur en 2012). Cette pénurie de moyens, comparée au nombre croissant d'affaires à traiter, se traduit donc sans surprise par un clearance rate d'environ $90 \%$ seulement.

Pour les conséquences économiques, sociales et psychologiques que cela induit, des durées de procédure trop longues représentent un coût évident pour la société. Cela peut même contribuer à la mise en cause de la crédibilité même du système judiciaire. En effet, les délais de jugement peuvent directement affecter un autre indicateur important de la qualité de la justice : la confiance des citoyens à l'égard de l'institution.

Dès lors que la Justice souhaite se recentrer sur ses « usagers ", la manière dont ceux-ci perçoivent l'institution judiciaire devient en effet un « élément constitutif de la qualité de celle-ci » (Bargues et Ferey, 2002, p. 194). Un sondage réalisé en mai 2008 par l'IFOP pour 
le Conseil supérieur de la magistrature révélait que $37 \%$ des personnes interrogées n'avaient pas confiance dans la justice 4 ( $45 \%$ en 2011 , selon un sondage IFOP pour le CSA). Comme détaillé dans le rapport du Conseil supérieur de la magistrature sur «les Français et leur justice » qui fait suite à ce sondage, les causes de la «crise de confiance » dans la Justice sont multiples. Elles sont à rechercher dans le décalage de plus en plus grand entre le caractère simple et concret des problèmes rencontrés par les usagers de la justice et la complexité et le dogmatisme attachés à l'institution judiciaire, dans l'évolution des rapports entre les pouvoirs exécutif, législatif et judiciaire ou encore dans l'évolution du rôle des magistrats dans la société. En termes de comparaison internationale, le European Value Study (EVS) offre une mesure de la confiance des citoyens à l'égard de la justice dans les 47 pays membres du Conseil de l'Europe (figure 2).

Figure 2. La confiance à l'égard de la Justice en 2008 (en pourcentage)

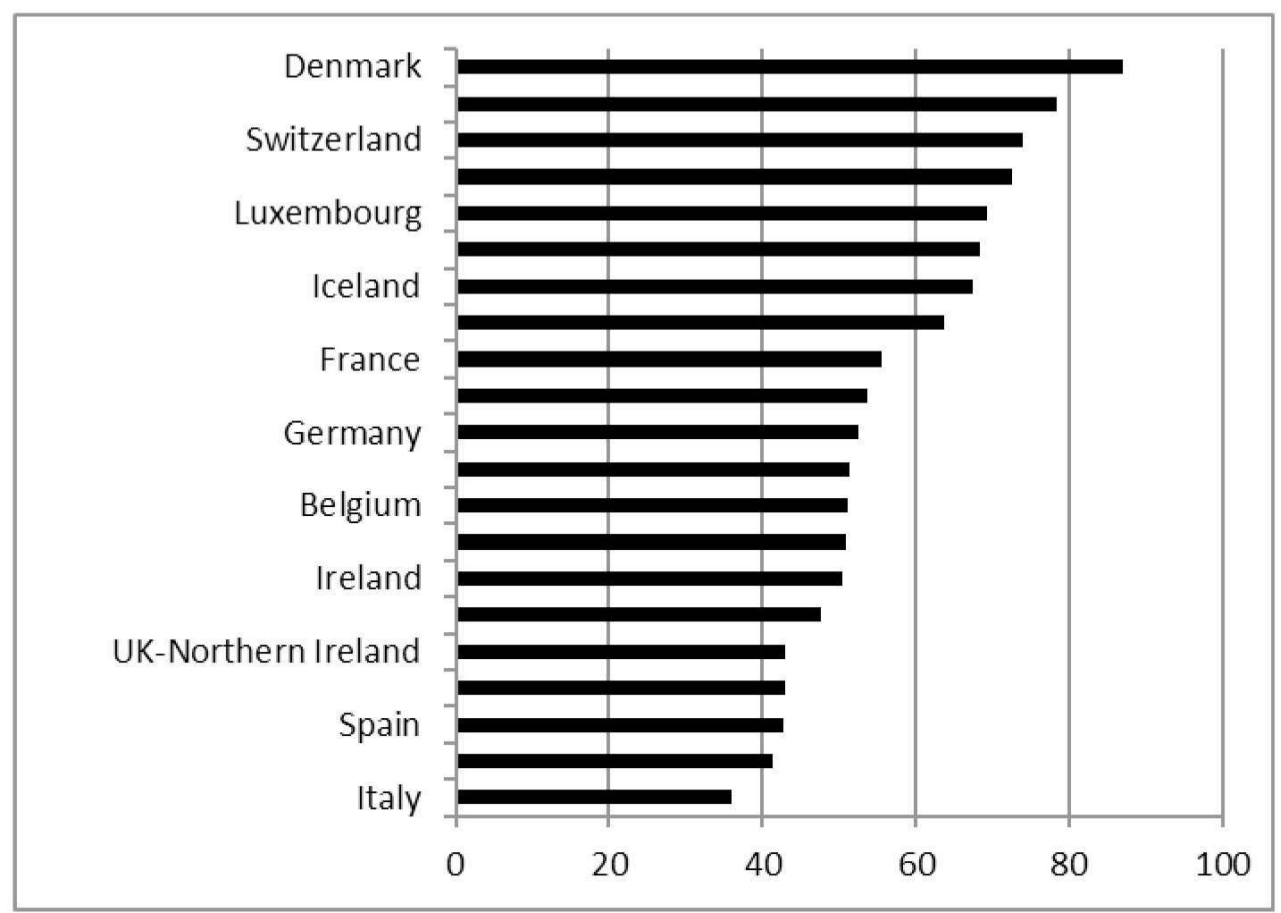

Source : European Value Study [2008]

19 En France, 55,5\% des personnes interrogées en 2008 déclaraient avoir «tout à fait confiance » ou " plutôt confiance » dans la Justice, soit beaucoup plus qu'en Italie ou en Espagne (respectivement $36,1 \%$ et $42,7 \%$ ) mais beaucoup moins qu'au Danemark ou en Norvège (respectivement $87,1 \%$ et $78,3 \%$ ).

20 Ainsi, le constat d'un déséquilibre sur le marché de LA justice a placé la question de la qualité au centre des préoccupations. Des discussions parlementaires, européennes et internationales sur l'organisation judiciaire ont été engagées afin de concevoir des techniques pertinentes d'évaluation de la justice, d'identifier les sources de blocage et potentiellement de guider des réformes permettant d'améliorer son fonctionnement.

21 Si ces discussions ont permis de mettre en évidence quelques aspects intéressants du problème, il semble qu'elles échouent cependant à fournir une analyse pleinement satisfaisante du système judicaire. 


\section{Analyse critique des programmes d'évaluation du fonctionnement des systèmes judiciaires}

Au début des années 2000, la logique de performance et l'idée de réformer la justice se propagent à plusieurs niveaux. En France, le Parlement vote en 2001 la Loi organique sur les lois de finances, qui vise à réformer la gestion de l'Etat et le management des agents publics ${ }^{5}$. En Europe, le Comité des Ministres du Conseil de l'Europe crée en 2002 la Commission Européenne pour l'Efficacité de la Justice (CEPEJ), qui se donne pour mission d'améliorer l'organisation et le fonctionnement des systèmes judiciaires européens. La même année, la Banque Mondiale lance le programme Doing Business, fournissant une évaluation de la « qualité » de la justice, entendue comme sa capacité à fournir des règles de droit assurant la protection des investisseurs. Les démarches de ces trois institutions apparaissent très différentes. Leurs approches de ce qu'est et de ce que doit être un système judiciaire le sont aussi. Pour autant, toutes restent parcellaires et biaisées car elles ne prennent en compte qu'imparfaitement les différentes missions de la justice.

\subsection{La LOLF : une dérive productiviste}

23 Afin de permettre une "meilleure efficacité de la justice », la LOLF vise à instaurer la transparence des choix budgétaires. Le passage d'une "culture des moyens» à une «culture des résultats » doit se faire en reliant l'attribution des crédits à une évaluation de la performance par rapport à des objectifs prédéfinis. Le lien entre les crédits accordés et les résultats doit théoriquement répondre à une logique incitative puisque des résultats jugés insatisfaisants sont "sanctionnés » par une diminution des crédits. La LOLF doit ainsi responsabiliser les personnels. Les dépenses de l'Etat sont désormais réparties par missions, qui correspondent aux grandes politiques de l'Etat.

La «mission justice» regroupe cinq «programmes»: la justice judiciaire, l'administration pénitentiaire, la protection judiciaire de la jeunesse, l'accès au droit et à la justice, le soutien de la politique de la justice et organismes rattachés. A chaque programme, sont associés : (i) un ensemble d'objectifs à atteindre pour répondre aux exigences d'une politique publique définie, (ii) un ensemble d'actions à poursuivre pour atteindre ces objectifs, (iii) des moyens nécessaires à la mise en œuvre des actions et (iv) des indicateurs permettant de mesurer et d'évaluer les résultats des actions menées. Un rapport annuel de performance doit rendre compte des résultats après avoir rappelé les objectifs et des coûts associés.

Depuis sa mise en œuvre en 2006, la LOLF a eu pour effet positif de favoriser l'introduction de nouveaux outils de gestion : on notera ainsi la mise en concurrence des prestataires de services et la mise en place de négociations tarifaires qui ont rendu possibles des économies budgétaires sensibles. De plus, une plus grande implication des acteurs a permis un changement des mentalités et des pratiques concernant la pertinence des frais engagés par rapport aux résultats attendus.

Toutefois, la mise en œuvre de la LOLF a suscité un certain nombre de débats et d'interrogations, relevés par Kirat (2010). Les inquiétudes se portent principalement sur la nature strictement quantitative des indicateurs de performance, ne parvenant pas, par conséquent, à refléter la réalité de l'activité judiciaire : « les syndicats de magistrats ainsi 
que le Sénat ${ }^{6} s^{\prime}$ inquiètent de l'aspect strictement quantitatif des indicateurs en raison de leur incapacité à refléter la réalité de l'activité judiciaire au sein des tribunaux » (Kirat, 2011, p. 5). D'ailleurs, pour l'auteur, l'un des défauts majeurs de ces indicateurs réside dans le fait que ce sont davantage des produits statistiques mesurant les flux d'entrée et de sortie que de véritables indicateurs de performance ou même de productivité: «la grande majorité des indicateurs ne sont pas des indicateurs de performance stricto sensu, mais s'appuient uniquement sur des données brutes quantitatives. (...) Seuls quelques indicateurs sont d'authentiques indicateurs de performance, entendue comme la productivité (moyenne) des juges ». (Kirat, 2010, p. 4 et 5)

Non seulement rendre compte de la qualité de la justice au regard de sa productivité est réducteur, mais cela peut générer des effets pervers: si les personnels sont incités à optimiser les variables quantitatives, cela peut se faire au détriment de la qualité (décisions moins bien motivées, plus d'erreurs de jugement...). Selon Didier Marshall, Premier président de la cour d'appel de Montpellier, la logique productiviste prévalant à la LOLF « ne permet pas (...) le travail de qualité qui répond à l'attente du justiciable ou à l'idée que le magistrat se fait de la qualité de sa décision» (Marshall, 2011, p. 20). En outre, pour juger des performances réalisées, les indicateurs calculés sont comparés à des valeurs-cible qui sont parfois jugées trop ambitieuses ou au contraire trop modestes ${ }^{7}$ et donc respectivement décourageantes ou désincitatives. Ainsi, Kirat (2010) note que vouloir fonder l'allocation des moyens des tribunaux sur des indicateurs biaisés, incomplets ou mal définis, et donc sujets à des interprétations erronées, peut conduire à compromettre l'efficacité et la qualité de la justice. C'est le cas par exemple lorsque de mauvais résultats, tels que mesurés par les indicateurs, se traduisent par une réduction des budgets alloués s'ils sont interprétés comme le reflet d'une mauvaise utilisation des moyens plutôt que comme un manque de moyens.

La mise en application de la LOLF suscite également des inquiétudes tant elle s'avère chronophage pour les personnels. Les magistrats, et principalement les chefs de cour, ont été amenés à s'investir davantage dans des activités de management et de gestion pour atteindre les objectifs définis et pour renseigner les indicateurs de performance. Ces nouvelles responsabilités ne s'accompagnent ni de moyens humains ou matériels supplémentaires, ni de formation adéquates et conduisent donc à restreindre leur temps consacré aux tâches juridictionnelles, d'où une augmentation des délais, une diminution du taux de résolution des affaires portées devant les tribunaux et une dégradation de la qualités.

29 Au total, la LOLF révèle une vision principalement productiviste du système judiciaire et laisse largement de côté tant les aspects qualitatifs que les aspects économiques et sociaux. Ces éléments permettent de douter de sa réelle capacité à promouvoir une amélioration de la «performance » ou de la «qualité » (au sens général du terme) du système judiciaire.

\subsection{La CEPEJ : une base de données riche encore faiblement exploitée}

En vue d'améliorer l'organisation et le fonctionnement des systèmes judiciaires des 47 Etats membres du Conseil de l'Europe, la CEPEJ a développé un outil d'évaluation approfondie destiné à identifier les forces et les faiblesses de chacun des systèmes judiciaires, pour proposer une orientation des réformes à engager. Les données recueillies 
par la CEPEJ sont à la fois qualitatives et quantitatives et couvrent tous les aspects du fonctionnement de la justice: le budget, sa répartition, l'information des usagers, l'équipement des tribunaux en nouvelles technologies de l'information et de la communication, le développement des modes alternatifs de résolution des litiges, les personnels judiciaires, leur mode de recrutement, leur formation, l'activité des tribunaux, etc.

31 Une telle base de données est d'autant plus précieuse que recueillir des informations homogènes et comparables dans des systèmes aussi hétérogènes n'est pas chose aisée. Cependant, la base statistique fournie reste sous-exploitée en termes de mesure de la performance des systèmes judiciaires européens. La mise en relation des «inputs » avec les « outputs » est limitée au calcul de deux indicateurs mesurant le taux de variation du stock d'affaires pendantes (clearance rate) et la durée estimée d'écoulement du stock d'affaires pendantes (disposition time). Ces deux indicateurs ont certes l'avantage d'être simples à construire et à interpréter, mais ils focalisent l'attention sur la productivité des systèmes. Or, la CEPEJ dispose d'éléments permettant d'apprécier d'autres aspects du fonctionnement de la justice, plus qualitatifs ou organisationnels, comme l'accès à la justice ou encore la formation des magistrats. Ces éléments mériteraient d'être mis en avant plus systématiquement. Si les travaux de la CEPEJ ont le mérite de décrire le fonctionnement des justices européennes ils ne permettent pas en revanche d'évaluer les systèmes judiciaires européens dans leur capacité à guider les comportements. Finalement, les bases de données de la CEPEJ, bien que recherchant l'exhaustivité, ne s'intéressent qu'aux résultats directs du fonctionnement de la justice et restent surtout largement sous-exploitées pour fournir une analyse pleinement satisfaisante des systèmes judiciaires.

\subsection{Doing Business : loin d'une évaluation de la performance globale de la justice, une représentation subjective de sa capacité à protéger les investisseurs}

32 Le programme Doing Business de la Banque Mondiale lancé en 2002 rassemble des données sur les normes et les procédures relatives à la règlementation des affaires et à la protection des droits de propriété dans 183 pays et anticipe leur impact en termes d'incitation pour les entrepreneurs. En 2011, l'évaluation porte sur onze critères économiques9. Les données sont collectées grâce à des questionnaires soumis à des professionnels de la finance, de l'économie et du droit. Elles sont destinées à la construction d'indicateurs thématiques, correspondants aux onze domaines étudiés, rassemblés ensuite en un indicateur global mesurant la "facilité de faire des affaires » dans chacun des pays. Les indicateurs ainsi créés sont utilisés pour établir des comparaisons entre pays sur la capacité des systèmes juridiques et judiciaires à favoriser l'entreprenariat.

La démarche de ce programme est intéressante en ce qu'elle consiste à évaluer le droit et la justice aux regards de leurs effets sur l'incitation à l'investissement. Cependant, depuis la publication du premier rapport en 2003, les faiblesses méthodologiques de Doing Business (Ménard et Du Marais, 2008) ainsi que la partialité de l'objectif retenu et ses conséquences en termes de politiques publiques ont souvent été soulignées (Du Marais, 2006). Elles sont aujourd'hui reconnues et admises, y compris par les initiateurs du programme. 
Tout d'abord, la construction des indicateurs de Doing Business repose sur des réponses individuelles à un questionnaire. La première remarque adressée aux auteurs du programme concerne la nature des résultats obtenus. En effet, la qualité du droit évaluée par la méthode des questionnaires ne repose pas sur un constat réel mais est largement fondée sur la perception des personnes interrogées. Il convient donc de bien garder à l'esprit dans toutes les interprétations, que les résultats de Doing Business relèvent d'une mesure subjective de la qualité du droit et non pas d'une mesure objective.

La seconde critique porte sur la conception des questions : celles-ci sont construites de manière à orienter les réponses vers la description des instruments juridiques permettant de résoudre un cas type. Selon les auteurs des rapports Doing Business, cela est nécessaire pour obtenir une mesure standardisée des institutions juridiques et judiciaires et ainsi faciliter les comparaisons internationales. Toutefois, comme rappelé dans Du Marais (2006), cette méthode des cas types revient à nier la diversité des solutions juridiques existantes pour résoudre le même problème dans les différents environnements institutionnels rencontrés. En conséquence, Doing Business reviendrait plus à mesurer l'écart à une norme prédéfinie arbitrairement par ses auteurs qu'à refléter la qualité réelle de la justice.

Enfin, le dernier problème que nous mentionnerons ici concerne le codage binaire des réponses. Le principe du codage suppose d'évaluer chacune des réponses en référence à un idéal prédéterminé. Mais le codage binaire ne permet pas de prendre en compte la multiplicité des objectifs des institutions juridiques et judiciaires. Les résultats sont donc inévitablement biaisés en faveur de l'objectif retenu. En focalisant leur attention sur la capacité du système à encourager l'investissement et la création d'entreprise, les auteurs de Doing Business sous-estiment l'importance des autres objectifs économiques et sociaux que la justice doit poursuivre. En guise d'illustration, les avantages de la flexibilité pour les investisseurs sont systématiquement reconnus tandis que la sécurité, la protection ou la prévisibilité juridique, bénéfiques à d'autres catégories d'agents (les employés par exemple), sont négligées. Enfin, l'évaluation porte uniquement sur le contenu des règles de droits en matière de protection des investisseurs et n'intègre pas les modes de mise en application de ces normes.

En pratique, le problème du programme Doing Business et de ses biais est que la portée de l'évaluation se veut clairement normative : les résultats des études statistiques visent à mettre en œuvre des réformes pour améliorer la qualité des systèmes telle que définie par le programme. Or, dans la mesure où les institutions juridiques et judiciaires ne sont approchées que de manière très partielle et discutable, tout jugement de valeur sur la performance globale de celles-ci est évidemment critiquable, et a fortiori, toute recommandation en termes de politiques économiques est sujette à caution.

Au total, les trois programmes que nous venons de présenter (LOFL, CEPEJ, Doing Business), bien que soulignant des dimensions importantes du rôle d'un système judiciaire, sont très incomplets. Avec la LOLF, les objectifs qualitatif et incitatif de la justice sont sousestimés; la CEPEJ reste focalisée sur la fonction de résolution des litiges; la Banque Mondiale ne s'intéresse qu'à la capacité de la justice à inciter les investisseurs et les entrepreneurs à " faire des affaires ", écartant de fait les effets incitatifs pour les autres acteurs et la capacité de la justice à résoudre les conflits de manière rapide et équitable. Finalement, aucune de ces approches, individuellement, ne parvient à intégrer l'ensemble des éléments à prendre en compte pour accéder à une bonne compréhension du 
fonctionnement d'un système judiciaire et de ses effets directs, sur la qualité de la justice et indirects, sur l'économie et la société.

\section{Proposition d'un cadre pour la compréhension du fonctionnement du système} une analyse pertinente du système judiciaire et de les présenter dans un outil de synthèse. rapide et équitable à la résolution des litiges. Le règlement des conflits est à envisager comme le résultat direct de l'activité juridictionnelle. Nous le qualifierons par la suite d' output de la justice. Ainsi, mesurer l'output revient à évaluer la justice dans sa capacité à donner aux agents les moyens d'agir pour corriger les externalités qu'ils subissent. Cela correspond à la fonction de réparation du système. (2) Inciter les agents économiques à de «bons » comportements, autrement, dit à des comportements susceptibles de favoriser le développement économique et social. Il s'agit là du résultat indirect de la justice en termes de retombées positives pour la société. Nous y ferons référence par le terme d' outcome. L'outcome permet d'évaluer l'efficacité du système dans sa fonction d'incitation ou encore de régulation des comportements économiques et sociaux. Bien sûr, l'output et l'outcome ne permettent pas directement d'apprécier l'efficacité de la justice. Pour ce faire, les résultats directs et indirects de l'activité judiciaire doivent être mis en relation avec les contraintes du système, c'est-à-dire la demande de justice et les moyens disponibles pour traiter les contentieux (i.e. l'offre de justice). Les résultats doivent également être appréciés au regard de l'organisation de la justice qui peut influencer l'efficacité pour des niveaux de demande et d'offre de justice donnés. Tous ces critères doivent être intégrés dans l'analyse du système judiciaire.

Du point de vue de l'output quantitatif, la justice doit faire face aux flux d'affaires dont elle est saisie pour éviter l'engorgement des juridictions. Les instruments de mesure de l' output quantitatif sont donc par exemple le nombre d'affaires terminées pendant une période de référence, le nombre de jugements exécutés ou encore le nombre d'affaires en stock au début de la période suivante. Mais polariser toute l'attention sur le seul aspect quantitatif de la justice fait prendre le risque de négliger d'autres aspects importants, moins faciles à appréhender, qui définissent l'output qualitatif. Ainsi, l'article 6 de la Convention européenne de sauvegarde des droits de l'homme et des libertés fondamentales pose les bases juridiques de l'exigence de qualité de la justice: «Toute personne a droit à ce que sa cause soit entendue équitablement, publiquement et dans un délai raisonnable, par un tribunal indépendant et impartial, établi par la loi, qui décidera, soit des contestations sur ses droits et obligations de caractère civil, soit du bien-fondé de toute accusation en matière pénale dirigée contre elle ». Dans l'énoncé de ce principe, figure les deux aspects à distinguer de l'output qualitatif de la justice: la qualité des procédures et la qualité des décisions rendues.

le plan de la qualité des procédures, le critère essentiel porte sur les délais. Deffains et Doriat-Duban (2001) montrent que sur le marché de la justice, ce sont les délais qui jouent spontanément le rôle de variable d'ajustement en cas d'excès de demande par rapport à l'offre, puisque les coûts de procès ne varient pas avec la demande. Dans l'idéal, les 
procédures se doivent d'être ni trop expéditives, ni trop longues. La durée souhaitable est donc fonction de la complexité et du type de contentieux. En outre, la qualité des procédures est un concept protéiforme qui englobe différentes dimensions d'accessibilité à la justice ${ }^{10}$. Sur le plan de la qualité des décisions, on s'attend à ce qu'une décision soit basée sur des critères objectifs, qu'elle soit motivée et pertinente au regard des règles de droit. De plus, les magistrats doivent rendre leurs décisions en toute indépendance, en particulier vis-à-vis du pouvoir politique.

Existe-t-il un arbitrage entre les deux « produits » de la justice, qualité et quantité ? Pour répondre à cette question, Chantrel et al. (2002) ont exploité des données qualitatives et quantitatives pour la France sur la période allant du début des années 1980 à la fin des années 1990. Ils montrent ainsi que l'augmentation du nombre d'affaires traitées sur cette période a impliqué une dégradation de la qualité, mesurée par la durée. Les années 1985-1989 font figure d'exception au milieu de cette tendance puisqu'un fort progrès technique a alors permis de "faire plus» tout en continuant à "faire mieux». Plus généralement, on peut songer aux effets pervers possibles si l'on tend à privilégier certains aspects de l'output de la justice au détriment des autres. Comme nous l'avons vu précédemment, il est par exemple souvent reproché à la LOLF de focaliser l'attention sur l'output quantitatif de la justice au risque de sacrifier l'output qualitatif. D'autre part, une politique judiciaire visant à réduire la longueur des procédures peut devenir expéditive et réduire la qualité des décisions rendues. Intuitivement, une amélioration simultanée des deux outputs est néanmoins possible si un accompagnement organisationnel est mis en œuvre ou si des moyens supplémentaires sont accordés.

Les résultats de l'activité de la justice ne sont pas seulement directs et interprétables en termes d'ouput, ils sont aussi indirects et interprétables en termes d'outcome. La notion d' outcome reflète l'idée que les règles et les pratiques judiciaires agissent comme des prix implicites qui orientent les choix des agents. Ces choix portent sur le caractère déviant ou non de leurs actions, sur leur participation à l'activité économique, mais aussi sur leur recours ou non au procès en cas de conflits. Par conséquent, nous allons définir ici deux types d'outcomes: l'outcome en termes d'incitations à de "bons" comportements économiques et sociaux et l'outcome en termes d'incitations à recourir à des modes de résolution des conflits extra-juridictionnels.

Tout d'abord, en élevant le coût des comportements déviants, la justice devrait inciter les agents à ne pas les adopter. Cela est valable à la fois en matière contractuelle et en matière criminelle (Posner, 1972; Becker, 1968). Ainsi, une justice idéale, dont les décisions et sanctions seraient prédictibles et crédibles, devrait avoir la capacité de faire disparaitre les contentieux par sa seule action incitative. Comme le note Asselain (2009): " comme toute action curative, l'action de la Justice vise idéalement à créer les conditions de sa propre inutilité ». De plus, l'analyse économique du droit suggère depuis Coase (1960) que si les agents sont parfaitement rationnels et la justice parfaitement prédictible et crédible, deux parties en conflits doivent être capables d'anticiper les gains qu'ils ont à éviter un procès et de prendre conscience qu'ils ont intérêts à s'arranger à l'amiable pour partager ces gains. Les échecs constatés de coopération renvoient alors en partie à l'incertitude juridique, c'est-à-dire à l'incapacité du système judicaire à assurer la prévisibilité et la sécurité des décisions de justice ${ }^{11}$.

Un autre aspect de l'outcome «socio-économique » de la justice concerne son impact sur la participation des agents à l'activité économique. Si les droits de propriété sont 
garantis, c'est-à-dire si la justice assure une sécurité économique aux agents, alors ceux-ci sont incités à investir, à entreprendre et à contribuer ainsi au dynamisme de l'économie.

Il est important de souligner que l'outcome de la justice est très dépendant de son output. Un système judiciaire capable de rendre des décisions rapidement et équitablement et de les faire appliquer est d'autant plus susceptible d'inciter les agents soit à adopter des comportements économiques ou sociaux désirables, soit à recourir à la négociation pour régler leurs différends.

Pour finir, la perception du système par les usagers apparait comme constitutive de sa qualité. Quelle que soit finalement la qualité réelle de la justice, si la qualité perçue est bonne, cela peut suffire en principe (au moins à court terme) à offrir une légitimité à l'institution, et à générer des retombées positives en termes d'outcome. Qu'elles concernent le comportement des agents dans la société ou leur comportement vis-à-vis de la justice, ces retombées sont vouées à réduire le nombre de contentieux entrants dans les tribunaux et donc à désengorger les juridictions tout en limitant le besoin de financement du système judiciaire.

L'efficacité de la justice ne s'apprécie pas uniquement au regard de l'output et de l'outcome mais en mettant en perspective ces résultats avec les contraintes du système à un instant donné. Les trois principales contraintes à envisager sont les moyens (l'offre), la demande et l'organisation du système judiciaire.

Les moyens correspondent aux ressources fournies par l'Etat : le budget des tribunaux ou du ministère public, le nombre de magistrats ou de fonctionnaires de justice (ou même au ratio fonctionnaires de justice / magistrats), l'équipement informatique des tribunaux ou encore les crédits alloués à l'aide judiciaire, etc. Mettre en relation l'output quantitatif avec les moyens revient à étudier la productivité de la justice, ou son coût moyen. Pour être efficace, un système judiciaire doit résoudre un maximum de contentieux sans erreurs, avec des moyens donnés.

51 La deuxième contrainte correspond à la demande de justice. Le volume de contentieux entrants dans les tribunaux et leur complexité déterminent la charge que le système judiciaire doit supporter. Plus la charge s'alourdit plus l'efficacité devient difficile à atteindre toute chose égale par ailleurs. Notons que la mise en relation de la demande avec les outputs permet de déterminer la réactivité du système judiciaire.

La troisième contrainte du système judiciaire porte sur son mode d'organisation. En adoptant des techniques efficaces de gestion, des dispositifs d'incitation des acteurs pertinents, des structures de décision adaptées etc., il est a priori possible de renforcer la productivité et la qualité de la justice toute chose égale par ailleurs.

Ainsi, l'analyse d'un système judiciaire ne peut se faire sans une mise en correspondance des résultats directs et indirects de la justice avec les contraintes du système, englobant à la fois les moyens disponibles, la demande de décisions de justice et son mode d'organisation. Par ailleurs, toute réforme visant à améliorer les résultats de la justice doit agir sur ses contraintes. Pour pouvoir anticiper l'impact de telles réformes, il faut donc pouvoir analyser les liens entre les contraintes et les résultats de la justice. Il convient également d'analyser les interconnections possibles entre les différentes contraintes pour anticiper les externalités positives ou négatives d'un changement. Autrement dit, analyser le système judiciaire reviendrait à expliciter et dynamiser le schéma de synthèse (figure 3) présenté ci-dessous. 
Figure 3. Cadre d'analyse du fonctionnement du système judiciaire

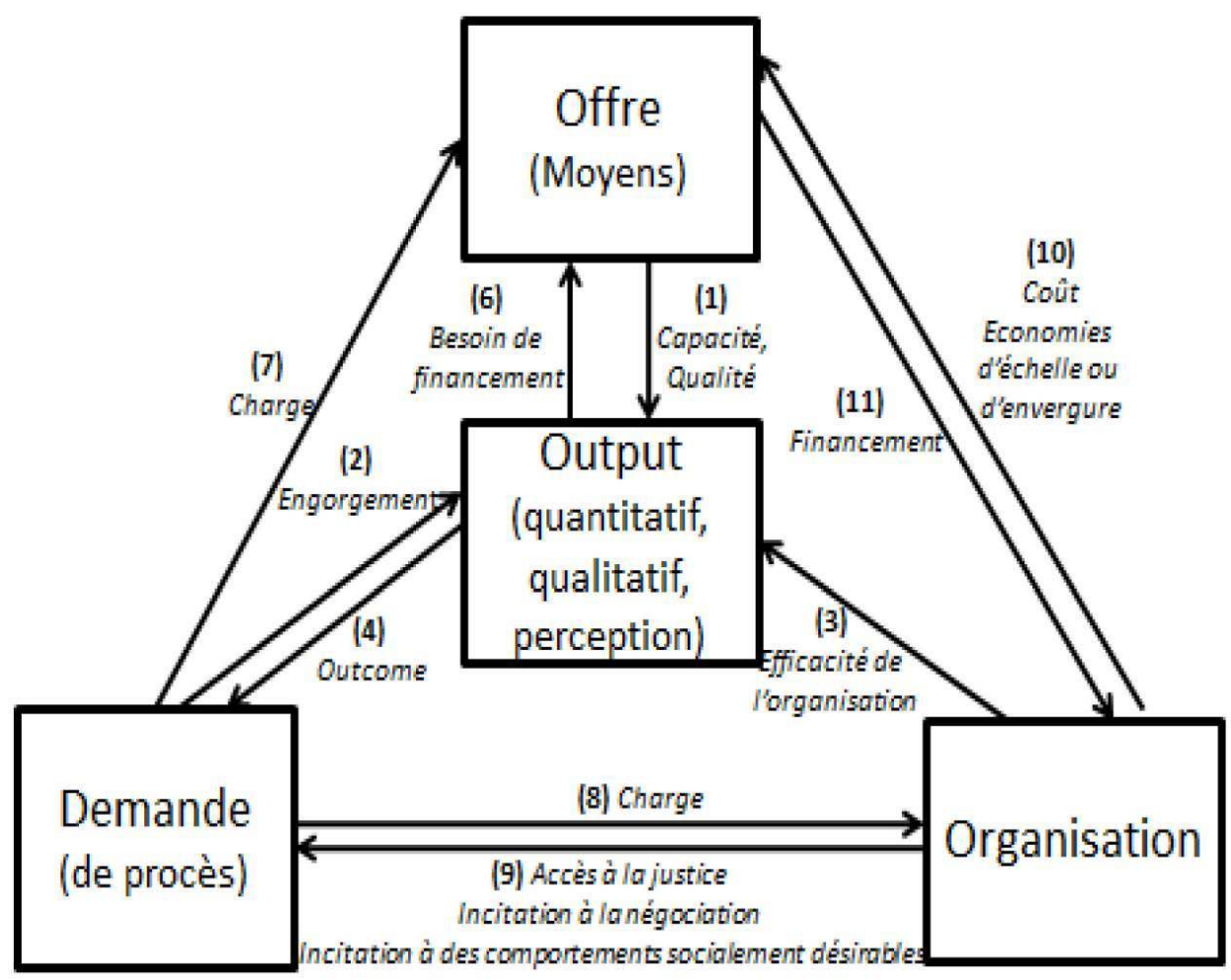

La section suivante a pour objet d'explorer ce cadre en proposant un regard général de la manière dont l'économie peut contribuer à l'analyse du fonctionnement du système judiciaire, au travers de contributions variées.

\section{Contribution de l'économie à l'analyse du fonctionnement du système judiciaire}

Au centre du schéma, l'output (l'engorgement des tribunaux, la qualité des affaires traitées, la confiance dans la justice) est la variable clé du système affectée par les trois contraintes que sont l'offre (les moyens) (1), la demande (2) et l'organisation (3). Par un effet indirect, en termes d'outcome, un changement dans l'offre ou l'organisation du système peut affecter la demande de décisions de justice (4). En nous référant à la littérature, nous pouvons envisager deux effets possibles d'une amélioration de l'output sur la demande.

- D’un côté, on peut imaginer qu'une amélioration de la qualité du système (réelle ou perçue) encourage le recours à la justice. En effet, une partie de la littérature, partant de l'hypothèse que les institutions formelles et informelles sont substituables (Kranton, [1996]), suggère que les agents ont davantage recours aux institutions formelles si celles-ci représentent une solution relativement plus efficace que les institutions informelles pour résoudre les conflits. Dans ces conditions, une amélioration de l'output accroit la demande de décisions de justice.

- En revanche, une autre partie de la littérature en économie du droit montre que si la justice est crédible, prédictible et fiable (par exemple si les juges sont indépendants et impartiaux), elle décourage les comportements opportunistes et facilite les arrangements à l'amiable en 
cas de conflit (Dari-Mattiacci et Deffains, 2007). Dans ce cas, si la qualité de la justice s'accroit, la demande de justice diminue. l'occurrence, l'économie du droit a déjà fourni quelques éléments d'analyse théorique qui confirment cette hypothèse et nous permettent d'ores et déjà de valider les relations entre l'offre du système judiciaire, l'un de ses outputs - les délais de jugement - et la demande de procès. En effet, Deffains et Doriat-Duban (2001) prouvent, grâce à un modèle théorique, que l'effet total d'un accroissement de l'offre de justice est ambigu compte tenu de son impact sur les délais de jugement des tribunaux. En effet, ils démontrent que si les affaires sont traitées plus rapidement, et que la demande de procès est élastique par rapport aux délais, le nombre d'affaires en attente de jugement peut augmenter à la suite d'une augmentation de l'offre. Cet effet pervers peut s'observer à court terme si la demande est suffisamment sensible aux délais. A long terme, il est encore plus probable, puisque la demande est toujours plus élastique à long terme qu'à court terme. Cette analyse nous enseigne que l'analyse d'une augmentation de l'offre sur la qualité du système ne doit pas omettre la prise en compte des effets indirects qui peuvent venir contrebalancer des conclusions a priori simples, voire simplistes, ne considérant que l'effet direct d'une telle mesure.

Du côté de l'analyse empirique, plusieurs auteurs ont cherché à spécifier l'effet des moyens du système judiciaire ou de son organisation sur son output qualitatif et/ou quantitatif ((1) et (3)). Kittelsen et Forsund (1992), ouvrent la voie à l'évaluation de l'efficacité des tribunaux grâce à la méthode de l'enveloppement des données (Data Envelopment Analysis ou DEA). L'intérêt de cette étude pionnière en matière de «jurimétrie $~^{12}$ est double puisque les auteurs se proposent à la fois de mesurer l'inefficacité relative des tribunaux en termes d'affaires traitées (donc d'output quantitatif) et de proposer des pistes d'amélioration de leur efficacité. A partir de données norvégiennes, ils mettent ainsi en évidence un excès de facteur travail dans les tribunaux les moins efficaces, pouvant s'expliquer par l'inefficience du capital technique utilisé. Ce travail reste l'une des premières mises en relation empiriques entre l'organisation des moyens de la justice (combinaison du facteur travail et du facteur capital) et ses résultats (3).

60 En 1999, Buscaglia et Dakolias contribuent à la littérature appliquée sur l'efficacité des systèmes judiciaires en offrant une étude comparative internationale de la performance des systèmes judiciaires, réalisée pour la Banque Mondiale. Ils y montrent l'impact des variables représentatives des moyens consacrés au système judiciaire ou de son 
organisation sur l'output du système judiciaire ((1) et (3)). Ils utilisent pour cela des données relatives aux tribunaux de commerces dans dix pays développés et en développement. Les variables retenues pour rendre compte de l'output du système judiciaire en matière de traitement des affaires commerciales sont au nombre de trois: les délais de traitement des affaires nouvelles, le taux de résolution des affaires nouvelles et l'élasticité du nombre d'affaires traitées par tribunal par rapport aux ressources budgétaires allouées (en quelque sorte la productivité des tribunaux). L'objet de cette étude est de montrer dans quelle mesure ces variables d'output peuvent s'expliquer par des variables de moyens - telles que le budget par tribunal, les ressources en capital fixe (infrastructures), les ressources en capital variables (nombre de juges et de greffiers, salaires des juges et ressources matérielles) - éclairant ainsi le lien (1) de notre cadre d'analyse, ou par des variables organisationnelles - comme l'utilisation des nouvelles technologies, le temps consacré par chaque juge aux activités juridictionnelles et administratives ou les techniques managériales - clarifiant alors le lien (3) du cadre d'analyse. Les résultats sont parfois surprenants dans la mesure où ils remettent en cause des idées largement ancrées dans les croyances collectives (celles des magistrats, des décideurs publics ou des citoyens) ${ }^{13}$. Par exemple, il ressort de cette étude que les délais de jugement s'expliquent plus par des facteurs organisationnels que budgétaires. Parmi les variables de moyens, seules les ressources en capital fixe (dans les infrastructures) sont susceptibles d'augmenter significativement le nombre d'affaires traitées par juge et de réduire la durée de traitement des affaires. En revanche, ni le niveau général du budget alloué aux tribunaux, ni le nombre de juges ou de greffiers, ni le salaire versé aux juges n'influencent ces deux variables d'output, contrairement à la perception des juges. Les auteurs notent cependant que si ces variables budgétaires ne présentent pas de lien statistique avec les variables d'output retenues pour leur étude (délais, taux de résolution des affaires, productivité des juges), elles peuvent influencer d'autres aspects de la qualité de justice, comme l'indépendance des magistrats par rapport au pouvoir politique ou le degré de corruption du système. Les variables organisationnelles affectant significativement l'output de la justice, d'après l'étude, sont l'utilisation des nouvelles technologies de l'information et de la communication, le partage du temps de travail des juges entre les activités juridictionnelles et administratives et les compétences managériales des juges.

61 Yeung et de Azevedo (2011) confirment en grande partie les résultats de Buscaglia et Dakolias sur la base de données brésiliennes. En particulier, ils montrent que les critiques régulières au Brésil consistant à pointer le manque de moyens humains et techniques comme facteur principal de l'inefficacité de certains tribunaux, sont infondées. Ils utilisent pour cela, tout comme Kittelsen et Forsund (1992), la méthode d'enveloppement des données, pour rendre compte des différences d'efficacité entre les différents tribunaux de l'analyse. Dans le même ordre d'idée que l'étude empirique de Buscaglia et Dakolias, leurs résultats tendent à prouver que l'inefficacité de certains tribunaux s'explique plus par un mauvais management des ressources que par leur manque.

Plus récemment, Schneider (2005) s'est intéressé à l'incidence d'autres facteurs organisationnels (le mode d'avancement des juges et leur niveau de qualification) sur l' output des tribunaux, éclairant un peu plus le lien (3) du cadre d'analyse. Dans un papier empirique, l'auteur analyse pour cela des décisions prononcées en appel par neuf Conseils des Prud'hommes allemands entre 1980 et 1998. L'output des tribunaux est mesuré à l'aide de deux variables : une variable quantitative, représentant la productivité des tribunaux ${ }^{14}$ 
et une variable qualitative (le taux de confirmation des décisions par la cour supérieure). L'auteur met ainsi en évidence deux résultats importants: d'une part, les tribunaux employant plus de juges hautement qualifiés (niveau doctorat) sont plus productifs mais leurs décisions sont moins souvent confirmées par la cour supérieure; d'autre part, les tribunaux employant des juges ayant été plus souvent promus par le passé sont moins productifs et prononcent des décisions qui sont moins souvent confirmées par la cour supérieure. Pour expliquer le second résultat, Schneider s'appuie sur les enseignements de la littérature théorique concernant l'effet incitatif des "tournois », initiée par Lazear et Rosen (1981). Selon cette littérature appliquée aux juges, il apparaît qu'au sein des tribunaux, la concurrence entre les juges les encourage à faire plus d'efforts pour s'assurer une promotion que seuls les plus performants obtiendront (Schneider, 2004). Dans un système où les juges sont nommés «à vie » et sont rémunérés par le biais de salaires fixes, les opportunités d'avancement ont ainsi un pouvoir incitatif potentiellement très important et sont donc susceptibles d'influencer la performance des juges, et par voie de conséquence, celle des tribunaux. De manière cohérente avec les résultats empiriques de Schneider, selon cette littérature théorique, le fait d'avoir au sein d'un « tournoi » (d'un tribunal) des juges qui se savent plus performants que les autres (et donc plus à même d'obtenir des promotions que les autres, car ayant obtenus plus de promotions par le passé) et que les moins performants repèrent également comme étant les plus performants qu'eux, réduit l'effet incitatif de la mise en place du tournoi. La raison en est que l'hétérogénéité des chances de succès a priori entre les membres du tournoi décourage l'effort à la fois des membres les moins performants (qui ne se reconnaissent qu'une chance infime de remporter le tournoi) et des plus performants (qui acquièrent un sentiment de supériorité "naturelle» sur les autres membres, les dispensant d'un effort). Schneider confirme ce résultat théorique par son évaluation empirique en montrant un output (quantitatif et qualitatif) statistiquement plus faible dans les tribunaux présentant une grande hétérogénéité de chances de promotion a priori entre leurs juges.

Comme nous l'avons vu précédemment, le lien entre l'offre et l'output de la justice (1) ne se réduit pas à l'impact des moyens sur les délais ou le taux de résolution des affaires ou ni même sur le taux de confirmation en appel ou en cassation. Un autre aspect de l'output concerne la confiance que le système judiciaire inspire aux individus. Logiquement, s'il existe un lien positif entre les moyens et l'output quantitatif et qualitatif, on peut avoir l'intuition qu'il existe aussi un lien positif entre les moyens et la confiance des citoyens, pour qui l'institution est plus crédible lorsque les délais sont raisonnables par exemple. Là encore l'économie du droit a permis d'aller au-delà de la simple hypothèse.

En particulier, Roussey et Deffains (2012) s'appuient sur une étude économétrique, croisant des données recueillies par la Commission Européenne pour l'Efficacité de la Justice avec des données du European Value Study, pour mettre en évidence l'existence d'un lien positif et significatif entre différentes variables budgétaires (budget, effectifs, intensité de l'aide judiciaire) et la confiance des citoyens à l'égard de leur système judiciaire. Cette corrélation positive entre les moyens de la justice et la confiance s'avère robuste à l'introduction de variables sociodémographiques et culturelles individuelles et de variables économiques et institutionnelles nationales. Ainsi, les auteurs testent l'hypothèse selon laquelle les ressources consacrées à la justice jouent un rôle de signal de la qualité du système et affectent donc positivement la perception des individus quand ceux-ci sont dans l'incertitude concernant le fonctionnement réel de l'institution. Par 
ailleurs l'impact du budget de la justice sur la confiance des individus apparait plus fort dans les pays où les individus déclarent être plus satisfaits du niveau de démocratie de leur pays. Ce résultat semble corroborer l'intuition selon laquelle un budget de la justice relativement élevé a d'autant plus d'effet sur la confiance des individus que ceux-ci ne craignent pas que les ressources consacrées à la justice soient détournées.

En pratique, une augmentation des moyens de la justice, dans le contexte actuel de crise économique et budgétaire, peut sembler difficilement réalisable. Lorsque l'urgence demande de consacrer des ressources à des secteurs directement affaiblis par la crise, la concurrence budgétaire se fait plus intense et le coût d'opportunité de la justice augmente. Toutefois, notre schéma d'analyse laisse entrevoir les externalités positives qu'une augmentation des budgets alloués à la justice peut induire. Si l'offre de justice augmente de manière à rendre la justice plus crédible aux yeux des agents et plus à même de garantir leurs droits de propriété (1), cela devrait se répercuter en termes d'incitations positives pour les agents, c'est-à-dire en termes d'outcome (4). Ils devraient être plus incités à investir et les co-contractants plus incités à respecter leurs engagements. Dès lors, une augmentation de l'offre de justice devrait non seulement avoir un effet direct sur la capacité des tribunaux à traiter les contentieux (1), mais aussi un effet indirect sur le nombre de contentieux lui-même (4) et donc, en retour, sur la charge pesant sur le système (2), (7), (8).

66 Cette intuition d'un effet multiplicateur des moyens de la justice a été récemment validée par Deffains et Roussey (2011) grâce à une analyse économique de la résolution des contentieux locatifs. Lorsque les tribunaux sont engorgés, la justice n'est plus en mesure d'assurer correctement la sécurité des propriétaires contre le risque d'impayé de loyer. En conséquence, les propriétaires adoptent des stratégies leur permettant de se protéger eux-mêmes contre ce risque. Parmi ces stratégies, la pratique d'un loyer élevé permet aux propriétaires de bénéficier d'une "prime de risque». Toutefois, celle-ci a pour effet pervers de précipiter les défauts de paiement et d'accroittre le nombre de conflits locatifs entrants dans les tribunaux. Ceci ne fait que renforcer le risque locatif pour les propriétaires... Les auteurs montrent grâce à la statique comparative, qu'une augmentation marginale des moyens de la justice consacrés à la résolution des conflits locatifs est à même d'améliorer de manière significative à la fois le fonctionnement des tribunaux et le fonctionnement du marché du logement locatif. En effet, dans un premier temps, par un effet direct, une augmentation des dépenses de justice permet d'augmenter la capacité des tribunaux et donc de réduire leur engorgement pour un nombre de cas entrants inchangé. L'amélioration du fonctionnement des tribunaux contribue à rassurer les propriétaires, qui réduisent la prime de risque incluse dans le montant des loyers. La diminution des loyers permet un relâchement de la contrainte budgétaire pesant sur les locataires et réduit ainsi le nombre d'impayés de loyer. Cet effet indirect est cumulatif puisque la diminution de la probabilité de défaut entraîne une diminution du nombre de litiges et donc une diminution additionnelle des délais de procédure, à capacité de traitement donné du système judiciaire. Les auteurs montrent ainsi que la demande de justice est indirectement affectée par l'offre de justice. Cette étude témoigne du fait que l'évaluation des retombées sociales d'une augmentation des ressources consacrées au système judiciaire doit nécessairement prendre en compte cette interdépendance entre l'offre et la demande.

67 L'organisation du système judiciaire peut avoir une incidence directe sur la qualité de celui-ci (3). L'économie du droit a permis de le démontrer grâce à l'analyse de plusieurs 
aspects des procédures judiciaires. Par exemple, les auteurs se sont intéressés à l'efficacité relative des différents "standards de preuve » quant à la minimisation des erreurs de jugement. Le standard de preuve correspond au degré de conviction requis pour trancher un litige. D’une manière générale, la littérature oppose la «prépondérance de la preuve", le standard de preuve le plus souvent requis dans les procès civils de Common Law et l'« intime conviction » du juge, concept plus flou retenu dans les pays de Droit Civil. La prépondérance de la preuve implique que le fait à juger soit considéré comme prouvé s'il est plus vraisemblable que son contraire, tandis que l'intime conviction exige un degré de conviction proche de la certitude (au-delà de tout doute raisonnable).

Les premières analyses comparatives du standard de preuve, comme celle de Brook (1982) ou Davis (1994) ont d'abord montré que la prépondérance de la preuve était plus à même de minimiser la probabilité d'erreurs de jugements que l'intime conviction. Cette conclusion repose sur le fait que si les erreurs de type I (trancher à tort contre le défendeur) et les erreurs de type II (trancher à tort contre le plaignant) représentent le même coût pour la société, la prépondérance de la preuve, qui répartit les erreurs de type I et de type II de manière symétrique, conduit à rejeter l'hypothèse la moins vraisemblable et donc à minimiser le coût espéré des erreurs. Mais, comme nous pouvons en avoir l'intuition grâce à notre outil de synthèse, l'effet du standard de preuve sur le système judiciaire ne se réduit pas à la qualité des jugements rendus (3). En particulier, le choix du standard de preuve n'est pas neutre vis-à-vis des incitations données aux agents économiques (9).

Demougin et Fluet $(2005,2006)$ par exemple se sont intéressés à cette question et ont ainsi pu nuancer les conclusions précédentes concernant le standard de preuve optimal. Grâce à des modèles théoriques de décision, ils ont pu analyser les conséquences du standard de preuve sur l'incitation des agents à adopter des comportements socialement désirables, par exemple la prévention du risque d'accident, Ils ont pu ainsi considérer l'incidence directe du standard de preuve sur la demande (7) et son incidence indirecte sur l'engorgement du système judiciaire (3). En termes d'efficacité globale du standard de preuve, les auteurs concluent ainsi à la nécessité d'arbitrage entre l'incitation à la prévention des accidents et la minimisation des erreurs.

Un autre effet à considérer dans l'analyse du standard de preuve est l'effet indirect de celui-ci sur la demande (3 puis 4). Car l'effet du standard de preuve sur la qualité des jugements, autrement dit sur les erreurs (3), est susceptible d'avoir des répercussions sur le comportement des individus (4). Le lien entre l'output qualitatif de la justice est la demande de procès (4) a été démontré par Polinsky et Shavell (1989). Plus précisément, les auteurs ont mis en évidence l'effet des erreurs judiciaires sur la décision des agents de recourir au procès ainsi que sur leur incitation à respecter la loi. Les conclusions de leur modélisation sont les suivantes : les erreurs de type II découragent les plaignants à avoir recours au procès car elles réduisent leur chance de victoire. Au contraire, les erreurs de type I encouragent au procès. De manière générale, les erreurs de jugement jouent positivement sur l'incitation des agents à respecter la loi parce qu'elles influencent à la fois la probabilité de procès et les résultats du procès. En supposant qu'un procès va effectivement avoir lieu, les deux types d'erreurs réduisent l'incitation des individus à respecter la loi : la probabilité qu'un individu n'ayant pas respecté la loi échappe à la sanction réduit son incitation à adopter un comportement socialement désirable tandis 
que la probabilité qu'un agent ayant respecté la loi soit malgré tout sanctionné réduit son incitation à bien se comporter car le gain relatif de cette action est réduite.

Un autre aspect de l'organisation de la justice n'est pas neutre vis-à-vis de la qualité de l'output du système judiciaire (3), du comportement des justiciables (8 et 4) et du cout imposé à la société (10). Il s'agit du degré d'initiative qu'il convient de laisser au juge dans la recherche des éléments de preuve. Ce point différencie également les procédures de Common Law, de type accusatoire et les systèmes de Droit Civil de type inquisitoire. Afin de déterminer quelle type de procédure est la plus efficace les auteurs en économie du droit ont mis en évidence les différents effets de chaque type de procédure. Dans la procédure accusatoire, les parties financent la production des preuves qu'elles recherchent elles-mêmes avant de les présenter au juge dont le rôle est relativement « passif ». Le coût public de la recherche de preuves est relativement faible dans ce cas. En revanche, dans la mesure où l'information des parties est imparfaite, celles-ci peuvent manipuler les preuves qu'elles présentent au juge, pour maximiser leur chance de victoire au procès, ce qui se révèle coûteux en termes de risque d'erreur de jugement. Dans la procédure inquisitoire, le juge est plus "actif », il dirige la procédure, convoque et interroge les témoins, désigne des experts. La procédure avec juge " actif » est coûteuse pour la société dans la mesure où elle est financée par des fonds publics. Cependant les preuves rassemblées, et donc les décisions de justice, sont de bonne qualité puisqu'elles dépendent de l'initiative du juge et non du jeu stratégique des parties. En définitive, les travaux théoriques les plus récents qui se sont penchés sur cette question semblent donc conclure à une relation inverse entre le coût de la procédure et la qualité des décisions qu'elle permet (Emons et Fluet, 2009). Ainsi un mode de production de production de preuve peut avoir à la fois un effet négatif en termes de coût de procédure (10) mais un effet positif sur la qualité des décisions (3). De manière intéressante, Deffains et al. (2007) introduisent un nouveau critère d'efficacité de la procédure, l'égalité d'accès au droit et à la justice (9), et nuancent ainsi les conclusions précédente jusqu'alors plutôt favorables à la procédure accusatoire.

Certaines modalités organisationnelles du système, en agissant directement sur le comportement économique des parties à un procès, permettent également de réguler l'accès à la justice (9). C'est le cas par exemple de la règle d'allocation des coûts de procès (Plott, 1987). Si les coûts de procès augmentent, le coût relatif du procès par rapport à un arrangement à l'amiable est également plus élevé. Cela a pour conséquence une baisse de la demande et finalement un meilleur traitement des affaires pour les agents ayant recours au procès grâce au désengorgement des tribunaux (2), ce qui compense l'effet négatif de la hausse des coûts de procès. Concernant les effets comparés des différentes règles d'allocation des coûts de procès rencontrées dans les systèmes judiciaires, l'analyse économique a montré que la règle américaine où chaque partie paie ses propres frais de procès est plus propice au règlement des litiges à l'amiable que la règle britannique dans laquelle le perdant se voit transférer la totalité des frais de procès du gagnant. Quant à la règle française de condamnation aux dépens, elle facilite davantage la négociation que la règle anglaise mais moins que la règle américaine.

73 Les modes alternatifs de résolution des litiges peuvent également contribuer au désengorgement des tribunaux (2) en favorisant le règlement à l'amiable des contentieux (9) (Shavell, 1995; Chappe, 2001). Selon la CEPEJ (2010), " les mesures alternatives au règlement des litiges (ADR - Alternative Dispute Resolution) peuvent contribuer à une hausse de l'efficacité de la justice en donnant aux citoyens des alternatives aux procédures judiciaires 
habituelles». Ces mesures, telles que la médiation ou la conciliation, reposent sur le volontariat des parties et requièrent l'intervention d'un tiers impartial dont le rôle est de communiquer des informations aux parties et de les aider à trouver un accord.

Intuitivement, les modes alternatifs de résolution des litiges peuvent contribuer non seulement à désengorger les tribunaux mais aussi à aboutir à des solutions satisfaisantes pour l'ensemble des parties. En économie, le procès est considéré comme un échec de coopération. En effet, dans un univers idéal, les individus devraient préférer négocier pour ensuite se partager le surplus correspondant à l'ensemble des frais d'instance économisés en évitant le procès. Des travaux théoriques et empiriques ont montré que des problèmes informationnels ou encore de répartition pouvaient être à l'origine des recours aux procès et que le taux d'arrangement augmentait avec le coût relatif du procès par rapport à la solution coopérative. Partant de là, les modes alternatifs de résolution des litiges apparaissent comme des solutions à la fois avantageuses financièrement par rapport au procès et capables de résoudre les problèmes informationnels faisant obstacle à la négociation des parties. En recourant à la théorie des contrats et l'économie de l'information, Chappe (2002) analyse également l'effet de l'arbitrage sur les incitations des agents à adopter des comportements permettant de réduire la survenance d'accident, et donc de litiges, lorsque l'arbitrage est décidé non pas après l'apparition d'un litige mais dans l'éventualité d'un litige au moment de la signature du contrat.

Ces travaux cités en exemple ci-dessus montrent la pertinence d'un raisonnement économique pour établir les directs et indirects des modalités organisationnelles du système judiciaire. Ils montrent que l'analyse économique peut contribuer utilement au débat sur les réformes du système judiciaire en anticipant les effets pervers ou vertueux de changements organisationnels ou budgétaires au sein de l'institution. Pour autant, ils n'épuisent pas le thème et il reste beaucoup à faire, tant du point de vue théorique qu'empirique, pour mettre à jour l'ensemble des liens directs et indirects existants entre les résultats et les contraintes du système judicaire.

\section{Conclusion}

Pour conclure, cet article nous a permis de montrer les limites des programmes existants pour guider les réformes nécessaires d'un système judiciaire en crise. Nous avons fourni un outil de synthèse pour dépasser une appréciation souvent incomplète ou partiale de l'activité de la justice. L'idée clé est que l'évaluation du système judiciaire doit prendre en compte non seulement les résultats directs de changements organisationnels ou budgétaires (par exemple, les délais de jugement ou les erreurs judiciaires) mais aussi les résultats indirects, beaucoup plus diffus, comme l'incitation des agents à adopter des comportements socialement désirables ou à négocier en cas de conflit. Nous avons montré que la science économique dispose des outils nécessaires à la mise en évidence de tels effets et qu'elle s'avère donc essentielle à une meilleure compréhension du fonctionnement du système judiciaire. Si les règles de droit intéressent les économistes depuis plusieurs dizaines d'années déjà, l'analyse économique du système judiciaire est à son commencement. L'outil de synthèse que nous proposons ici peut être utile pour guider les recherches futures sur ce thème. 


\section{BIBLIOGRAPHIE}

Asselain J.-C., (2009), L'argent de la justice. Le budget de la justice en France de la Restauration au seuil du XXIe siècle, Bordeaux, Presses Universitaires de Bordeaux, $551 \mathrm{p}$.

Bargues C. et S. Ferey, (2002), « Les référents de l'image publique de la justice » dans E. Breen (sous la direction de), Evaluer la justice, Presses Universitaires de France, p. 175-195.

Becker G. S., (1968), « Crime and punishment: An economic approach », Journal of Political Economy , vol. 76(2), p. 169-217.

Brook J., (1982), « Inevitable errors: The preponderance of evidence standard in civil litigation », Tulsa Law Journal, vol. 18, p. 89-105.

Buscaglia E. et M. Dakolias, (1999), « Comparative international study of court performance indicators: a descriptive and analytical account », Washington, DC: World Bank, 50p.

http://documents.worldbank.org/curated/en/1999/08/728946/comparative-internationalstudy-court-performance-indicators-descriptive-analytical-account

Commission Européenne pour l'Efficacité de la Justice, (2014), Rapport d'évaluation des systèmes judiciaires européens de la CEPEJ - Edition 2014 (données 2012), Les Etudes de la CEPEJ n² 20, Conseil de l'Europe.

Commission Européenne pour l'Efficacité de la Justice, (2012), Rapport d'évaluation des systèmes judiciaires européens de la CEPEJ - Edition 2012 (données 2010), Les Etudes de la CEPEJ nº 18, Conseil de l'Europe.

Commission Européenne pour l'Efficacité de la Justice (2010), Systèmes judiciaires européens (données 2008) : efficacité et qualité de la justice, Les Etudes de la CEPEJ n 12, Conseil de l'Europe.

Commission Européenne pour l'Efficacité de la Justice (2008), Systèmes judiciaires européens (données 2006) : efficacité et qualité de la justice, Les Etudes de la CEPEJ n 11, Conseil de l'Europe. Commission Européenne pour l'Efficacité de la Justice (2006), Systèmes judiciaires européens (données 2004) : efficacité et qualité de la justice, Les Etudes de la CEPEJ n 1, Conseil de l'Europe Chantrel E., R. Giraud et S. Guibaud, (2002), « Quelques pistes d'analyse économique », dans E. Breen (sous la direction de), Evaluer la justice, Presses Universitaires de France, p. 240-273.

Chappe N., (2001), «L'analyse économique d'un mode de résolution des litiges : l'arbitrage », Revue Française d'Economie, vol. 15(4), p. 187-208.

Chappe N., (2002), « The informational role of the arbitration clause ", European Journal of Law and Economics, vol. 13(1), p. 27-34.

Coase R.H., (1960), « The problem of social cost », Journal of Law and Economics, vol. 3, p. 1-44.

Dari-Mattiacci G. et B. Deffains, (2007), « Uncertainty of law and the legal process », Journal of Institutional and Theoretical Economics, vol. 163(4), p. 627-656.

Davis M.L., (1994), « The value of truth and the optimal standard of proof in legal disputes ", Journal of Law, Economics, and Organization, vol. 10(2), p. 343-359.

Deffains B., D. Demougin et C. Fluet, (2007), « Economie des procédures judiciaires », Revue Economique, vol. 58(6), p. 1265-1290. 
Deffains B. et M. Doriat-Duban, (2001), « Equilibre et régulation du marché de la justice. Délais versus prix », Revue Economique, vol. 52(5), p. 949-974.

Deffains B. et L. Roussey, (2011), « The multiplier of public expenditure on justice: The case of rental litigation ", Review of Law and Economics, vol. 7(1), p. 249-269.

Deffains B., (2007), « Introduction à l'analyse économique des systèmes juridiques ", Revue Economique, vol. 58(6), p. 1149 à 1162.

Demougin D. et C. Fluet, (2005), « Deterrence versus judicial error: A comparative view of standards of proof », Journal of Institutional and Theoretical Economics, vol. 161(2), p. 193-206.

Demougin D. et C. Fluet, (2006), « Preponderance of evidence », European Economic Review, vol. 50 (4), p. 963-976.

Du Marais B., (2006), Des indicateurs pour mesurer le droit? Les limites méthodologiques des rapports Doing Business, Paris, La Documentation Française, 153 p.

Emons W. et C. Fluet, (2009), « Accuracy versus falsification costs: The optimal amount of evidence under different procedures ", Journal of Law, Economics, and Organization, vol. 25(1), p. 134-156.

European Values Study, (2008). 4th wave, Integrated Dataset, GESIS Data Archive, Cologne, Germany, ZA4800 Data File Version 2.0.0 (2010-11-30) doi:10.4232/1.10188 (http:// dx.doi.org/10.4232/1.10188).

Jean J.-P., (2010), « Etude de 16 pays comparables », CEPEJ, Conseil de l'Europe, http://www.coe.int/t/dghl/cooperation/cepej/evaluation/2010/2010_pays_comparables.pdf

Kirat T., (2010), « Performance-based budgeting and management of judicial courts in France: an assessment ", International Journal for Court Administration, $\mathrm{n}^{\circ}$ 2, p. 1-9.

Kittelsen S.A.C. et F.R. Forsund, (1992), « Efficiency analysis of Norvegian district courts ", Journal of Productivity Analysis, vol. 3, p. 277-306.

Kranton R.E., (1996), « Reciprocal exchange: A self-sustaining system », American Economic Review, vol. 86(4), p. 830-851.

Lazear E.P. et S. Rosen, (1981), « Rank-order tournaments as optimum labor contracts », Journal of Political Economy, vol. 89, p. 841-864.

Marshall D. (2011), « Les tribunaux à l'heure de la performance » dans B. Frydman et E. Jeuland (sous la direction de), Le nouveau management de la justice et l'indépendance des juges, Dalloz, p. 11-21.

Menard C. et B. Du Marais, (2008), « Can we rank legal systems according to their economic efficiency? ", Journal of Law and Policy, vol. 26, p. 55-80.

Plott C.R., (1987), « Legal fees: A comparison of the American and English rules ", Journal of Law, Economics, and Organization, vol. 3(2), p. 185-192.

Polinsky A. M. et S. Shavell, (1989), «Legal error, litigation, and the incentive to obey the law », Journal of Law, Economics and Organization, vol. 5(1), p. 99-108.

https://ideas.repec.org/a/oup/jleorg/v5y1989i1p99-108.html

Roussey L. et B. Deffains, (2012), « Trust in judicial institutions: An empirical approach », Journal of Institutional Economics, vol. 8(3), p. 351-369.

Schneider M. R, (2004), « Careers in a judicial hierarchy », International Journal of Manpower, vol. 24, p. 431-446. 
Schneider M. R, (2005), « Judicial career incentives and court performance: An empirical study of the German labour courts of appeal », European Journal of Law and Economics, vol. 20(2), p. 127-144.

Shavell S., (1995), « Alternative dispute resolution: An economic analysis », Journal of Legal Studies, vol. 24(1), p. 1-28.

Yeung L.L. et P.F. de Azevedo, (2011), « Measuring efficiency of Brazilian courts with data envelopment analysis (DEA) », IMA Journal of Management Mathematics, vol. 22(4), p. 1-14.

\section{NOTES}

1. Particulièrement depuis la mise en place de la loi d'orientation et de programmation pour la justice (LOPJ) en 2002.

2. Autriche, Belgique, Danemark, Finlande, Italie, Luxembourg, Pays-Bas, Norvège, Pologne, Portugal, Espagne, Suède, Suisse, Angleterre et Pays de Galle, Ecosse.

3. Source : Ministère de la Justice.

4. Soit un plus petit pourcentage que pour la défiance à l'égard des élus (55\%) et des médias (69\%) mais plus grand que pour la défiance à l'égard de la fonction publique (27\%), la police (24\%), l'armée (18\%), l'école (18\%) et les hôpitaux (11\%).

5. Nous étudions ici le cas de la Loi organique sur les lois de finance (LOLF) en France, mais l'on peut également citer, comme expérience nationale de mesure de la performance le cas du projet de Trial Courts Performance Standards aux Etats-Unis, ou du programme pour le renforcement de la qualité de la justice aux Pays-Bas par exemple.

6. «Les indicateurs du projet annuel de performance consacré à la mission "Justice" sont (...) construits selon une approche essentiellement quantitative, qui ne peut donner qu'une idée très partielle et grossière de l'activité judiciaire ", Sénat, Projet de loi de finances pour 2011 : Justice et accès au droit.

7. Asselain (2009) donne à cet égard des exemples d'objectifs se situant en-deçà des performances déjà atteints dont le taux-cible d'évasion sous garde pénitentiaire directe, fixé à 3,4 pour 10000 détenus en 2010 alors que le taux constaté en 2006 est de 1,9.

8. Kirat (2010) fait référence à plusieurs études empiriques mettant en évidence une corrélation entre la charge administrative des juges et plusieurs indicateurs de performance ou d'efficacité de la justice. En particulier : Buscaglia et Dakolias (1999) dans une étude comparative basée sur des données judiciaires provenant de 10 développés et en développement, répartis sur trois continents : la France, l'Ukraine, la Hongrie, Singapour, le Panama, le Chili, le Pérou, l'Equateur, l'Argentine et la Colombie ; Yeung et de Azevedo (2009) sur des données brésiliennes et Kittelsen et Forsund (1992) sur des données norvégiennes. Nous détaillons ces études dans la section 5.

9. Création d'entreprise, obtention des permis de construire, transfert de propriété, obtention de prêts, protection des investisseurs, paiement des taxes et impôts, commerce transfrontalier, exécution des contrats, fermeture d'entreprise, raccordement au réseau électrique, embauche des travailleurs.

10. L'accès aux bâtiments, la sécurité des usagers, l'accueil des usagers par des personnels courtois, à l'écoute, compréhensifs ; l'accès à l'information concernant les procédures, l'orientation, la transparence ; et enfin l'accès financier, grâce à l'aide judiciaire, incluant, entre autres mesures garantissant l'égalité de traitement devant la justice, la représentation par un professionnel de justice.

11. L'autre explication renvoyant à la rationalité imparfaite des agents.

12. Le terme est employé par Buscaglia et Dakolias (1999) pour faire référence aux statistiques et à l'économétrie appliquées à l'évaluation du système judiciaire. 
13. Buscaglia et Dakolias confrontent d'ailleurs de manière très intéressante les résultats de leur étude empirique avec la perception des juges quant au lien entre les différentes variables budgétaires ou organisationnelles et celles mesurant l'output.

14. La productivité est ici approchée par un score obtenu à partir de la méthode d'enveloppement des données incluant le nombre de cas terminés pendant l'année relativement au nombre de juges et au nombre des affaires à traiter et le nombre de décisions publiées dans un registre.

\section{RÉSUMÉS}

La justice subit une crise qui se manifeste par de nombreux dysfonctionnements. Les réformes permettant d'améliorer l'efficacité de la justice doivent être guidées par une analyse fine du système judiciaire. Or, les principaux programmes d'évaluation des systèmes judiciaires menés en France et à l'étranger restent pour l'heure insatisfaisants pour envisager l'ensemble des effets d'une réforme organisationnelle ou budgétaire. A l'aide d'une représentation synthétique du système judiciaire, nous montrons que la science économique dispose des outils permettant de mettre en évidence les effets directs et indirects d'une réforme et ainsi de contribuer à la réflexion sur une amélioration du fonctionnement de la justice.

The judiciary suffered from a crisis that manifests itself by many failures. Reforms aimed at improving the efficiency of justice need to be guided by a careful analysis of the judicial system. However, evaluation programs conducted in France and abroad are insufficient to consider all the effects of an organizational or budgetary reform. Using a systemic approach of the judiciary, we show that economic analysis provide tools to highlight both direct and indirect effects of a reform and can thus contribute to the debate about improvement of the judiciary functioning.

\section{INDEX}

Mots-clés : système judiciaire, analyse économique, réformes organisationnelles

Keywords : judicial system, economic analysis, output, outcome, constraints, analytical framework

Code JEL K4 - Legal Procedure; the Legal System; and Illegal Behavior

\section{AUTEUR}

\section{LUDIVINE ROUSSEY}

Université Paris Descartes, Sorbonne Paris Cité, LIRAES 